\title{
Bris de retraitement des dispositifs médicaux dans une clinique de médecine familiale d'Ottawa, en Ontario, en 2018
}

\author{
Geneviève Cadieux ${ }^{1 *}$, Dara Spatz Friedman ${ }^{1}$, Leslie Tilley ${ }^{1}$, Tony Mazzulli² ${ }^{2}$ Cameron McDermaid ${ }^{1}$
}

\section{Résumé}

Contexte : En avril 2018, Santé publique Ottawa a identifié plusieurs manquements aux pratiques de prévention et de contrôle des infections $(\mathrm{PCl})$ à grande échelle, dont un bris de retraitement des dispositifs médicaux (RDM) critiques réutilisables, sur une période de quinze ans dans une clinique de médecine familiale.

Objectifs : Décrire l'intervention de santé publique et estimer le risque de transmission du virus de l'hépatite $B(\mathrm{VHB})$, du virus de l'hépatite $C(\mathrm{VHC})$ et du virus de l'immunodéficience humaine $(\mathrm{VIH})$ en lien avec ce bris de RDM.

Méthodes : Les patients qui ont subi un acte préoccupant (lors duquel un dispositif médical critique réutilisable aurait pu être utilisé) à cette clinique ont été identifiés à l'aide des données du Régime d'assurance-santé de l'Ontario, et avisés individuellement. Les tests de dépistage du VHB, VHC et VIH ont été réalisés par le laboratoire de Santé publique Ontario et le risque d'infection a été estimé.

Résultats : Parmi les 4495 patients possiblement exposés à ce bris de RDM, 1496 (33,3\%) ont subi un dépistage dans les six mois qui ont suivi l'avis. La prévalence de l'infection au VHB, $\mathrm{VHC}$ et $\mathrm{VIH}$ dans ce groupe était inférieure à celle de la population canadienne en général. Parmi les patients d'abord diagnostiqués avec le VHB après un acte préoccupant, les risques de transmission du VHB n'étaient pas plus élevés lorsque l'acte s'était déroulé dans les 7 ou 28 jours après un autre patient avec un résultat positif pour le $\mathrm{VHB}\left(\mathrm{RC}_{7 \text { jours, ajusté selon l'âge }}=0,59\right.$, IC de $95 \%: 0,14$ à 2,51; $R C_{28 \text { jours, ajusté selon lâge }}=1,35, \mathrm{IC}$ de $95 \%: 0,62$ à 2,93). II n'a pas été possible d'estimer les risques de transmission du VHC et du VIH puisqu'aucun patient n'a été diagnostiqué avec le VHC ou le VIH après avoir subi un acte préoccupant dans les 28 jours après un autre patient avec un résultat positif pour le $\mathrm{VHC}$ ou le $\mathrm{VIH}$.

Conclusion : Aucune évidence de transmission du VHB, VHC ou VIH associée au bris de RDM n'a été constatée. Cependant, on ne peut pas complètement exclure la possibilité d'une transmission, puisque seulement le tiers des patients possiblement exposés ont subi les tests de dépistage.
Cette oeuvre est mise à la disposition selon les termes de la licence internationale Creative Commons Attribution 4.0

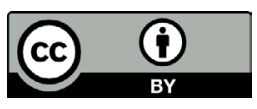

Affiliations

${ }^{1}$ Santé publique Ottawa, Ottawa, ON (lorsque cette étude a été menée)

${ }^{2}$ Laboratoire de Santé publique Ontario et Département de biopathologie et de médecine de laboratoire, Université de Toronto, Toronto, ON ${ }^{\star C}$ Correspondance : genevieve.
cadieux.ccsmt|@ssss.gouv.qc.ca

Citation proposée : Cadieux G, Friedman DS, Tilley L, Mazzulli T, McDermaid C. Bris de retraitement des dispositifs médicaux dans une clinique de médecine familiale d'Ottawa, en Ontario, en 2018. Relevé des maladies transmissibles au Canada 2020;46(2/3):45-53. https://doi.org/10.14745/ccdr.v46i23a04f

Mots-clés : prévention et contrôle des infections, manquement/transgression en matière de contrôle des infections, milieux de soins de santé communautaire, exercice privé, normes privées, médecine familiale, interventions chirurgicales ambulatoires, prévention et contrôle de la contamination croisée, contamination de l'équipement

\section{Introduction}

Dans la période de dix ans allant de 2008 à 2017, les Centers for Disease Control and Prevention (CDC) des États-Unis ont noté 61 éclosions dues aux virus de l'hépatite $B(\mathrm{VHB})$ et
$\mathrm{C}(\mathrm{VHC})$ liées à des écarts entre les pratiques observées et les pratiques exemplaires en matière de prévention et de contrôle des infections $(\mathrm{PCl})$ dans les établissements de soins (1). Plus de 115000 patients potentiellement exposés ont été avisés dans le cadre de ces enquêtes sur les éclosions associées aux soins 
de santé et 179 cas de VHB et plus de 295 cas de VHC ont été identifiés ( 1 ). La majorité ( $n=58 / 61$ ou $95 \%$ ) de ces éclosions de VHB et VHC associées aux soins de santé est survenue dans un milieu de soins extrahospitalier (1). Malheureusement, des données de surveillance nationale semblables ne sont pas disponibles pour le Canada; par conséquent, on ignore le fardeau des infections au VHB et au VHC associées aux manquements aux pratiques de $\mathrm{PCl}$ et aux bris de retraitement des dispositifs médicaux à l'échelle canadienne. Cependant, une enquête récente des unités de santé publique de l'Ontario a permis de constater une hausse de presque six fois les plaintes relatives aux pratiques de $\mathrm{PCl}$ et presqu'un triplement des manquements aux pratiques de $\mathrm{PCl}$ de 2015 à 2018 (2).

Les objectifs du présent article sont : 1) décrire un manquement aux pratiques de $\mathrm{PCl}$ à grande échelle incluant un bris de retraitement des dispositifs médicaux (RDM) critiques réutilisables dans une clinique de médecine familiale d'Ottawa, en Ontario; 2) estimer les risques de transmission du VHB, du $\mathrm{VHC}$ et du virus de l'immunodéficience humaine $(\mathrm{VIH})$ découlant de ce bris de RDM et 3 ) illustrer les défis rencontrés lors de l'intervention de santé publique en lien avec ce bris de RDM.

\section{Contexte}

En Ontario, le mandat et l'organisation des unités de santé publique sont définis par la Loi sur la protection et la promotion de la santé (3). À I'heure actuelle, l'Ontario possède 35 unités de santé publique : 21 indépendantes d'administrations municipales locales, sept départements de santé publique régionaux et sept unités de santé publique liées à l'administration d'une municipale à pallier supérieur ou à une autre administration municipale (4). Les Normes de santé publique de l'Ontario (5) définissent les programmes et services de santé publique obligatoires et les protocoles et lignes directrices connexes fournissent un encadrement supplémentaire. En 2015, le ministère de la Santé et des Soins de longue durée de l'Ontario a modifié le Protocole concernant les plaintes relatives aux pratiques de prévention et de contrôle des infections (6), qui demande aux unités de santé publique d'enquêter sur les pratiques de $\mathrm{PCl}$ dans un éventail de milieux, dont ceux des services personnels ( $p$. ex. salons de manucure, de coiffure et de tatouage) et les établissements au sein desquels les membres d'une profession de la santé réglementée ( $p$. ex. infirmiers, médecins, dentistes) mènent leurs activités.

Conformément au Protocole concernant les plaintes relatives aux pratiques de prévention et de contrôle des infections, 2019 (6), les unités de santé publique doivent recevoir les plaintes relatives aux pratiques de $\mathrm{PCl}$, enquêter sur ces plaintes et prendre des mesures visant à réduire le risque d'infection. En général, à la suite d'une plainte, un inspecteur ou infirmier de santé publique réalise une inspection des lieux au moyen des outils de vérification et autres ressources développés par Santé publique Ontario (7) et le Comité consultatif provincial des maladies infectieuses $(8,9)$ dans le but d'évaluer les écarts entre les pratiques observées et les pratiques exemplaires en matière de $\mathrm{PCl}$. Dans le Protocole concernant les plaintes relatives aux pratiques de prévention et de contrôle des infections, on définit le manquement aux pratiques de $\mathrm{PCl}$ comme le « non-respect des pratiques de prévention et de contrôle des infections, entraînant ainsi un risque de transmission de maladies infectieuses aux clients, aux personnes présentes ou aux membres du personnel par l'exposition de ces personnes à du sang, à des liquides organiques, à des sécrétions, à des excrétions, à des muqueuses, à des lésions cutanées ou encore à du matériel contaminé et à des articles souillés » (6). La plupart des plaintes relatives aux pratiques de $\mathrm{PCl}$ enquêtées par les unités de santé publique révèlent des écarts entre les pratiques observées et les pratiques exemplaires en matière de $\mathrm{PCl}$ qui, suite à une évaluation du risque, ne représentent pas un risque suffisant de transmission d'infections pour qu'il s'agisse d'un manquement ou d'un bris. Santé publique Ontario offre du soutien aux unités de santé publique en lien avec les évaluations du risque complexes.

Si le médecin hygiéniste ou une personne désignée détermine qu'il y a eu un manquement aux pratiques de $\mathrm{PCl}$, il doit le déclarer publiquement sur le site Web de l'unité de santé publique, en vertu du Protocole concernant les déclarations relatives aux pratiques de prévention et de contrôle des infections (10). Si un exploitant (c.-à-d. une personne qui exploite un milieu de service personnel ou de soins de santé) ne collabore pas à l'enquête ou ne met pas les mesures correctives en place dans les délais négociés et si le médecin hygiéniste ou un inspecteur en santé publique est d'avis qu'il existe un risque pour la santé, une ordonnance en vertu de l'article 13 conformément à la Loi sur la protection et la promotion de la santé peut être émise pour faire cesser une pratique ou la prestation d'un service, ou encore fermer un milieu de services personnels ou de soins.

\section{Méthodes}

\section{Détection du manquement aux pratiques de prévention et du contrôle des infections}

Santé publique Ottawa (SPO) a reçu une plainte d'un membre du public concernant la propreté d'une clinique de médecine familiale, y compris son équipement médical, en avril 2018. Le personnel de SPO a inspecté la clinique le même jour que la plainte a été reçue. On a découvert plusieurs écarts entre les pratiques de la clinique et les pratiques exemplaires en $\mathrm{PCl}$, dont : 1) l'ensemble des étapes de retraitement des dispositifs médicaux critiques réutilisables, 2) I'entreposage et l'administration des médicaments, 3) l'entreposage et la manipulation des spécimens à être analysés au laboratoire, 4) I'hygiène des mains, 5) le nettoyage environnemental, 6) les pratiques de base et précautions additionnelles et 7) la santé et la sécurité au travail. Ces écarts entre les pratiques observées 
et les pratiques exemplaires en matière de $\mathrm{PCl}$ semblaient avoir été présents depuis l'ouverture de la clinique en décembre 2003, jusqu'au moment de la plainte en avril 2018. II convient de noter que les médecins sont membres d'une profession autoréglementée et il n'y a pas d'inspection périodique des pratiques de $\mathrm{PCl}$ dans les cliniques médicales extrahospitalières de l'Ontario.

La clinique s'est prêtée volontairement à l'exigence de SPO de cesser de réaliser les actes médicaux nécessitant l'usage de dispositifs médicaux critiques réutilisables jusqu'à nouvel ordre. Plusieurs inspections de suivi ont permis d'assurer le respect des mesures correctives exigées par SPO.

\section{Évaluation du risque}

Conformément au mandat de santé publique en vertu de la Loi sur la protection et la promotion de la santé, l'évaluation du risque s'est penchée sur la probabilité de transmission du $\mathrm{VHB}, \mathrm{VHC}$ et $\mathrm{VIH}$, puisque ces infections peuvent échapper au diagnostic pendant plusieurs années, menant ainsi à des conséquences néfastes sur la santé et une transmission secondaire. Une interrogation du Système intégré d'information sur la santé publique (SIISP) pour les cas déclarés de VHB, VHC et $\mathrm{VIH}$ résidant dans la zone desservie par la clinique n'a pas permis d'identifier d'excès de cas comparativement au reste d'Ottawa ou au Canada.

Santé publique Ontario a évalué de manière qualitative le risque de transmission d'infections associé au bris de retraitement des dispositifs médicaux critiques réutilisables comme étant «faible » pour le VHB et le VHC, et " très faible " pour le VIH. Santé publique Ontario a recommandé le rappel des patients et de dépistages du VHB, le VHC et le VIH.

De plus, SPO a effectué une évaluation quantitative du risque au moyen d'une méthodologie publiée dans les écrits scientifiques $(11,12)$. L'évaluation du risque de SPO s'est basée sur des estimations de la prévalence du VHB (13), VHC (14) et VIH dans la population canadienne (15), des estimations du risque de transmission du VHB, du VHC et du VIH après une exposition percutanée publiées dans les écrits scientifiques (16) et a supposé le pire scénario, soit que la stérilisation était totalement inefficace; les résultats de cette évaluation (non publiés) étaient comparables à l'évaluation qualitative de Santé publique Ontario. Si le risque estimé avait été plus près du seuil de 1:1 000000 à partir duquel le rappel des patients est préconisé dans les écrits scientifiques $(11,12)$, le cadre d'éthique de 2014 de SPO aurait également pu être utilisé pour guider le processus décisionnel concernant le rappel des patients, comme cela a été le cas lors de l'intervention de SPO suite au bris de RDM à une clinique d'endoscopie en 2011 (17).

\section{Patients possiblement exposés : Définition}

Un patient possiblement exposé était défini comme une personne ayant subi un acte médical nécessitant possiblement l'usage d'un dispositif médical critique réutilisable à la clinique de médecine familiale concernée entre son ouverture en décembre 2003 et la cessation de ces actes en avril 2018 selon les données de facturation du Régime d'assurance-santé de I'Ontario (RASO). Les patients étaient considérés « possiblement exposés " (plutôt qu'exposés) puisqu'il se pourrait que certains actes n'aient pas nécessité l'usage de dispositifs médicaux critiques réutilisables ( $p$. ex. une réparation de lacération faite avec de la colle au lieu de sutures).

\section{Patients possiblement exposés : Identification}

Plus de 90000 patients uniques ont été traités entre décembre 2003 et avril 2018. Pour identifier les patients possiblement exposés à un dispositif médical critique réutilisable, SPO a révisé les types de procédures invasives effectuées à la clinique et évalué si des dispositifs médicaux critiques jetables à usage unique ou réutilisables étaient utilisés. Conformément à I'information obtenue de la clinique, SPO a conclu que les actes préoccupants étaient les suivants : ablation des acrochordons, grains de beauté et kystes avec un bistouri ou des ciseaux; biopsie cutanée; incision, drainage et tamponnement d'un abcès ou kyste; ablation d'un ongle incarné; réparation de lacération; retrait de sutures ou d'agrafes; retrait d'un corps étranger.

En collaboration avec la clinique, SPO a produit une liste des codes de facturation correspondant aux actes préoccupants. Ensuite, la division du Régime d'assurance-santé du ministère de la Santé et des Soins de longue durée de l'Ontario a extrait et transmis à SPO toutes les facturations soumises par les médecins de la clinique en lien avec l'un des actes préoccupants pour lesquelles le code postal était le même que celui de la clinique (il n'y avait qu'une clinique dans ce code postal à six caractères).

\section{Patients possiblement exposés : Rappel}

Les patients possiblement exposés à la suite du présent bris de RDM ont été avisés par la poste en juillet 2018. La lettre d'avis a été approuvée par SPO et envoyée par la clinique. Le jour de l'envoi postal, SPO a tenu une conférence de presse et donné des entrevues aux médias pour divulguer l'information aux patients possiblement exposés qui n'auraient pas été identifiés grâce aux données de facturation ( $p$. ex. patients non assurés, services non assurés, omissions dans la facturation). SPO a également publié un site Web avec de l'information détaillée sur ce bris de RDM le jour de l'envoi postal. Ce même jour, SPO a envoyé une télécopie aux partenaires du système de soins de santé, y compris les fournisseurs de soins primaires, pour les informer du bris de RDM, du rappel et du dépistage recommandé pour les patients, ainsi que pour fournir des ressources concernant le counseling des patients, les analyses de laboratoire à demander et le suivi clinique. Puisque la décision de subir les dépistages est une décision individuelle, des rappel additionnels (au-delà de la lettre d'avis) n'ont pas été envoyés aux patients. SPO a participé à de nombreuses entrevues avec les médias locaux concernant le taux de participation aux dépistages et les résultats agrégés plusieurs semaines et mois après l'envoi initial des lettres d'avis aux patients. 


\section{Facilitation des dépistages}

Une demande d'analyse du laboratoire de Santé publique Ontario (LSPO) pour le dépistage du VHB, du VHC et du VIH préremplie par l'un des médecins de la clinique accompagnait chaque lettre d'avis, permettant aux patients de se rendre directement à un centre de prélèvements pour une prise de sang et d'éviter une consultation médicale visant à obtenir une ordonnance pour des analyses de laboratoire.

Les patients qui ne souhaitaient pas que leurs résultats soient envoyés à la clinique ont été invités à consulter le professionnel de la santé de leur choix dans le but d'obtenir une ordonnance pour des analyses de laboratoire. Un formulaire d'ordonnance du LSPO partiellement rempli (avec les analyses à demander et le numéro d'enquête spéciale) a été publié sur le site Web de SPO, afin que les patients puissent l'apporter au professionnel de santé de leur choix.

\section{Analyses de laboratoire}

Le LSPO a réalisé toutes les analyses pour le VHB, le VHC et le $\mathrm{VIH}$ en lien avec le rappel, et un numéro d'enquête spéciale a permis de suivre tous les résultats en lien avec le rappel. Le dépistage sérologique initial pour le $\mathrm{VHB}$, le $\mathrm{VHC}$ et le $\mathrm{VIH}$ a été réalisé à l'aide de l'instrument Architect d'Abbott (Abbott Laboratories, Wiesbaden, Allemagne) en respectant les directives du fabricant. Les échantillons de sérum positifs pour les anticorps du VHC ont ensuite été analysés avec une deuxième analyse sérologique (ORTHO ${ }^{\circledR}$ HCV Version 3.0 ELISA Test System, Ortho Clinical Diagnostics Inc., Raritan, New Jersey, États-Unis), tandis que les échantillons de sérum positifs aux anticorps du $\mathrm{VIH}$ ont été ensuite analysés à l'aide du dépistage Geenius ${ }^{\mathrm{TM}}$ HIV 1/2 Confirmatory Assay (Bio-Rad Laboratories, Redmond, Washington, États-Unis). Les patients pour lesquels l'analyse sérologique suggérait une infection ont ensuite subi une analyse et un génotypage de I'ADN/ARN dans le cadre du suivi clinique habituel (cobas ${ }^{\circledR} \mathrm{HBV}$ et cobas $^{\circledR} \mathrm{HCV}$ avec I'instrument Roche 6800, Roche Molecular Systems Inc., Laval, Québec, Canada; Abbott RealTime HIV-1 en utilisant le système m2000 d'Abbott, Abbott Molecular Inc., Des Plaines, Illinois, États-Unis).

Si un agrégat d'au moins deux cas de $\mathrm{VHB}$, de $\mathrm{VHC}$ ou de $\mathrm{VIH}$ du même génotype avait été détecté, nous aurions demandé au Laboratoire national de microbiologie de procéder à d'autres analyses moléculaires. Le LSPO a transmis tous les résultats d'analyses disponibles à SPO un mois, trois mois et six mois après le rappel des patients. De plus, le LSPO a transmis à SPO tous les résultats positifs et négatifs des analyses moléculaires et sérologiques pour le VHB, VHC et $\mathrm{VIH}$ depuis 1996, pour les patients avec un dépistage positif pour le $\mathrm{VHB}, \mathrm{VHC}$ ou $\mathrm{VIH}$ ayant été possiblement exposés à la suite du bris de RDM. Ces données ont été utilisées pour estimer le moment auquel l'infection est probablement survenue, ainsi que la période infectieuse possible.

\section{Enquêtes des cas de $\mathrm{VHB}, \mathrm{VHC}$ et $\mathrm{VIH}$}

Les infections à $\mathrm{VHB}, \mathrm{VHC}$ et $\mathrm{VIH}$ sont des maladies à déclaration obligatoire en Ontario en vertu de la Loi sur la protection et la promotion de la santé. À ce titre, les nouveaux cas déclarés à SPO sont tous enquêtés conformément aux politiques et procédures en vigueur. Spécifiquement, un infirmier de santé publique communique avec le médecin demandeur du test, lui demande de remplir un formulaire de suivi et de l'envoyer à SPO; ce formulaire demande de l'information sur les facteurs de risque, la prise en charge clinique et la recherche des contacts effectué par le professionnel de la santé; de plus, le formulaire fourni un outil pour le counseling des patients quant aux mesures pour éviter la transmission. Au besoin, un infirmier de santé publique communique également avec le cas ou son plus proche parent pour obtenir les facteurs de risque, coordonner la prise en charge clinique, effectuer la recherche de contacts et offrir du counseling. Les renseignements recueillis sont saisis dans le SIISP.

Les patients possiblement exposés au bris de RDM avec des résultats positifs pour le $\mathrm{VHB}, \mathrm{VHC}$ ou $\mathrm{VIH}$, diagnostiqués suite au rappel ou avant le rappel et signalés à SPO, ont tous été enquêtés selon la méthode susmentionnée. Ensuite, l'infirmière-gestionnaire et l'épidémiologiste de l'équipe des infections transmissibles sexuellement et par le sang de SPO ont passé en revue manuellement les dossiers de tous les cas de VHB, VHC et VIH possiblement exposés à la suite du bris de RDM afin d'évaluer la présence d'agrégats ou de transmission associées au bris de RDM, et d'identifier les autres facteurs de risque.

\section{Analyses statistiques}

La prévalence des infections au VHB, VHC et VIH a été estimée chez les patients ayant subi une analyse après le rappel.

$L^{\prime}$ association entre la survenue d'un diagnostic d'infection au VHB, VHC ou VIH après un acte préoccupant et cet acte ayant eu lieu dans les sept jours suivant un autre acte concernant un patient avec un résultat d'analyse positif pour le même virus antérieur à leur propre acte a été estimée à l'aide de rapports de cotes. L'évaluation de l'exposition et du résultat clinique était fondée sur les résultats d'analyse de laboratoire disponibles et les dates des actes préoccupants. Le résultat clinique était défini comme l'état d'infection au VHB, VHC ou VIH après l'acte préoccupant. Aux fins de la présente analyse, les patients étaient considérés exposés lorsqu'ils avaient subi au moins un acte préoccupant dans les sept jours suivant un patient avec un résultat de dépistage positif pour le $\mathrm{VHB}$, le $\mathrm{VHC}$ ou le $\mathrm{VIH}$ antérieur à leur propre acte. Les patients étaient considérés non-exposés lorsqu'ils avaient subi un acte préoccupant plus de sept jours après un patient avec un résultat de dépistage positif pour le VHB, le VHC ou le VIH antérieur à leur propre acte ou après un patient sans résultat de dépistage positif pour le VHB, le VHC ou le VIH. La fenêtre de sept jours utilisée était fondée sur les données concernant la survie du virus (18-22) 
et la fréquence d'utilisation et de retraitement des dispositifs médicaux critiques réutilisables (c'est-à-dire que la transmission n'a été considérée que pour la première personne sur laquelle le dispositif mal stérilisé a été utilisé; les cycles de retraitement ultérieurs devraient avoir réduit le risque de transmission à nul).

Étant donné que les infections au VHB et au VHC peuvent se résoudre spontanément, nous ne pouvions pas déterminer de manière définitive l'état d'infection au VHB et au VHC de certains patients au moment de leur acte. Par exemple, on considérait un patient sans analyse négative antérieure pour l'hépatite $B$ ayant d'abord obtenu un résultat positif pour les anticorps anti$\mathrm{HBc}$ et négatif pour les antigènes $\mathrm{Hbs}$ (indiquant une immunité site à une infection naturelle résolue) après le rappel comme une source potentielle d'infection pour un autre patient ayant subi un acte préoccupant dans une fenêtre de sept jours, et comme ayant potentiellement été infecté lors de son propre acte préoccupant. Puisque l'information sur la charge virale du VIH n'était pas accessible (c'est-à-dire que les résultats d'analyses pour la charge virale du VIH n'ont pas à être déclarés à la santé publique en Ontario), les patients avec un dépistage positif du $\mathrm{VIH}$ avant leur acte préoccupant étaient considérés contagieux au moment de leur acte.

Le rapport de cotes a été la mesure de l'association choisie parce que la faible participation au dépistage n'a pas permis une estimation fiable de la prévalence des infections et les intervalles de confiance de $95 \%$ ont été estimés à l'aide de la méthode exacte. Les analyses ont été effectuées à l'aide de la version 14.2 du logiciel Stata (StataCorp, États-Unis). Des analyses de sensibilité ont été effectuées avec une fenêtre de temps plus courte et plus longue (un et 28 jours) et avec les données au niveau des actes-patients plutôt qu'au niveau des patients.

\section{Résultats}

Un total de 4595 patients ayant subi un acte préoccupant ont été identifiés à partir des données de facturation du RASO (figure 1); ensemble, ces patients ont subi 6832 actes préoccupants. Parmi ces 4595 patients, 28 ont communiqué avec SPO après avoir reçu une lettre d'avis pour indiquer qu'ils n'avaient jamais subi d'acte préoccupant à la clinique et 78 n'ont jamais fréquenté la clinique selon les dossiers de la clinique. Six autres patients non-identifiés à partir des données de facturation du RASO ont communiqué avec SPO pour indiquer avoir subi un acte préoccupant à la clinique. Parmi le total révisé de 4495 patients possiblement exposés, 1496 (33,3\%) ont subi le dépistage recommandé pour le $\mathrm{VHB}$, le $\mathrm{VHC}$ ou le $\mathrm{VIH}$ au moins une fois dans les six mois ayant suivi l'envoi postal des lettres $d^{\prime}$ avis concernant le bris de RDM.

En moyenne, les patients ayant subi le dépistage recommandé étaient plus âgés que ceux n'ayant pas subi le dépistage. La participation au dépistage recommandé ne différait pas selon le moment de l'acte préoccupant (récemment ou il y a plusieurs années) ou le nombre d'actes préoccupants (tableau 1).

\section{Figure 1 : Identification de patients possiblement exposés à ce bris de retraitement des dispositifs médicaux critiques réutilisables}

Patients possiblement exposés identifiés à partir des données du RASO
$\begin{aligned} & \text { Patients ayant affirmé n'avoir jamais subi d'acte préoccupant et } \\ & \text { ayant reçu la lettre de notification par erreur }\end{aligned}$

Abréviations : RASO, Régime d'assurance-santé de l'Ontario; VHB, virus de l'hépatite B; VHC, virus de l'hépatite $\mathrm{C}$; VIH, virus de l'immunodéficience humaine

Remarque : Certains patients pourraient avoir reçu une lettre, déterminé n'avoir jamais subi d'acte préoccupant et omis d'aviser Santé publique Ottawa ou la clinique; ces patients sont compris dans ce chiffre comme étant possiblement exposés. Certains patients pourraient ne pas avoir reçu de lettre, s'être considérés comme ayant subi un acte préoccupant et avoir cherché à se faire dépister sans utiliser la demande d'analyse de laboratoire spéciale contenant le numéro de suivi d'enquête; ces patients ne sont pas compris dans ce chiffre

Tableau 1 : Caractéristiques des patients possiblement exposés au bris de retraitement des dispositifs médicaux critiques réutilisables, selon la participation au dépistage du VHB, VHC et VIH six mois après l'envoi de la lettre d'avis $(N=4495)$

\begin{tabular}{|c|c|c|c|c|c|c|}
\hline \multirow[t]{2}{*}{$\begin{array}{c}\text { Caractéristique } \\
\text { du patient }\end{array}$} & \multicolumn{3}{|c|}{$\begin{array}{l}\text { Patients possiblement } \\
\text { exposés qui ont subi un } \\
\text { dépistage } \\
(N=1496)\end{array}$} & \multicolumn{3}{|c|}{$\begin{array}{l}\text { Patients possiblement } \\
\text { exposés qui n'ont pas } \\
\text { subi un dépistage } \\
\text { ( } N=2999)\end{array}$} \\
\hline & \multicolumn{2}{|c|}{$\mathrm{N}$} & $\%$ & \multicolumn{2}{|c|}{$\mathrm{N}$} & $\%$ \\
\hline \multicolumn{7}{|l|}{ Groupe d'âge } \\
\hline 0 à 17 ans & & 142 & 10 & & 274 & 9 \\
\hline 18 à 44 ans & & 410 & 27 & & 1414 & 47 \\
\hline 45 à 64 ans & & 566 & 38 & & 884 & 30 \\
\hline 65 ans et plus & & 377 & 25 & & 426 & 14 \\
\hline $\begin{array}{l}\text { Données } \\
\text { manquantes }\end{array}$ & & 1 & $\begin{array}{r}\text { moins } \\
\text { de } 1\end{array}$ & & 1 & $\begin{array}{r}\text { moins } \\
\text { de } 1\end{array}$ \\
\hline \multicolumn{7}{|c|}{ Moment de l'acte préoccupant le plus récent } \\
\hline $\begin{array}{l}\text { Il y a moins de } \\
12 \text { mois }\end{array}$ & & 144 & 10 & & 198 & 7 \\
\hline $\begin{array}{l}\text { Entre } 12 \text { et } 23 \\
\text { mois }\end{array}$ & & 138 & 9 & & 256 & 9 \\
\hline $\begin{array}{l}\text { Entre } 24 \text { et } 35 \\
\text { mois }\end{array}$ & & 125 & 8 & & 209 & 7 \\
\hline $\begin{array}{l}\text { Il y a } 36 \text { mois } \\
\text { ou plus }\end{array}$ & & 1089 & 73 & & 2336 & 78 \\
\hline \multirow{2}{*}{$\begin{array}{l}\text { Nombre d'actes } \\
\text { « risqués » }\end{array}$} & Moyenne & Médiane & Étendue & Moyenne & Médiane & Étendue \\
\hline & 1,5 & 1 & 1 à 25 & 1,6 & 1 & 1 à 23 \\
\hline
\end{tabular}

Parmi les patients ayant subi un dépistage du VHB, VHC ou $\mathrm{VIH}$ après l'envoi des lettres d'avis, il y a eu deux nouveaux diagnostics d'infection au VHC (tableau 2) : un patient avec un ARN positif (indiquant une infection chronique) et un patient avec un ARN négatif (indiquant une infection résolue). Le patient avec une infection chronique au $\mathrm{VHC}$ a été référé à un 
Tableau 2 : Nombre de patients ayant subi et $n$ 'ayant pas subi un acte préoccupant dans les sept jours suivant un patient potentiellement infectieux, par résultat de dépistage du VHB, VHC et VIH six mois après l'envoi des lettres d'avis $^{\mathrm{a}}$

\begin{tabular}{|c|c|c|c|c|c|c|c|c|}
\hline \multirow[b]{2}{*}{ État d'exposition } & \multicolumn{3}{|c|}{$\begin{array}{l}\text { Résultat d'analyse pour le } \mathrm{VHB}^{\mathrm{b}} \\
\qquad(\mathrm{N}=1466)\end{array}$} & \multicolumn{3}{|c|}{$\begin{array}{l}\text { Résultat d'analyse pour le } \mathbf{V H C}^{\mathrm{b}} \\
\qquad(\mathrm{N}=1441)\end{array}$} & \multicolumn{2}{|c|}{$\begin{array}{c}\text { Résultat d'analyse } \\
\text { pour le VIH } \\
(\mathrm{N}=1251)\end{array}$} \\
\hline & $\begin{array}{l}\text { Infection } \\
\text { chronique }\end{array}$ & $\begin{array}{l}\text { Infection } \\
\text { résolue } \\
\text { (immunité }^{\text {naturelle) }}{ }^{c}\end{array}$ & $\begin{array}{l}\text { Non } \\
\text { infecté }\end{array}$ & $\begin{array}{c}\text { Positif } \\
\text { à l'ARN } \\
\text { (infection } \\
\text { chronique) }\end{array}$ & $\begin{array}{c}\text { Positif } \\
\text { pour les } \\
\text { anticorps, } \\
\text { négatif } \\
\text { à l'ARN/ } \\
\text { inconnu }\end{array}$ & $\begin{array}{l}\text { Non } \\
\text { infecté }\end{array}$ & Infecté & $\begin{array}{l}\text { Non } \\
\text { infecté }\end{array}$ \\
\hline $\begin{array}{l}\text { A subi un acte préoccupant dans les } \\
\text { sept jours }{ }^{d} \text { suivant un autre patient avec } \\
\text { un résultat d'analyse positifd pour le } \\
\text { même virus antérieur à son propre acte } \\
\text { (exposé) }\end{array}$ & 0 & 2 & 153 & 0 & 0 & 78 & 0 & 8 \\
\hline $\begin{array}{l}\text { N'a pas subi d'acte préoccupant dans } \\
\text { les sept jours }{ }^{d} \text { suivant un autre patient } \\
\text { avec un résultat d'analyse positif pour le } \\
\text { même virus antérieur à son propre acte } \\
\text { (non exposé) }\end{array}$ & 1 & 26 & 1284 & 5 & 12 & 1346 & 1 & 1242 \\
\hline
\end{tabular}

Abréviations: $\mathrm{VHB}$, virus de l'hépatite $\mathrm{B} ; \mathrm{VHC}$, virus de l'hépatite $\mathrm{C} ; \mathrm{VIH}$, virus de l'immunodéficience humaine

Ce ne sont pas tous les patients ont subi un dépistage des trois agents infectieux; par conséquent, les totaux des cellules sont différents pour chaque infection

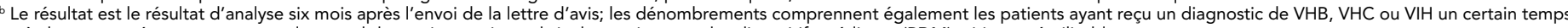
après leur acte préoccupant et avant le rappel des patients suite au bris de retraitement des dispositifs médicaux (RDM) critiques réutilisables

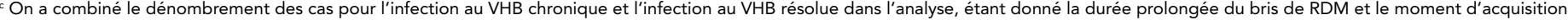
inconnu de l'infection

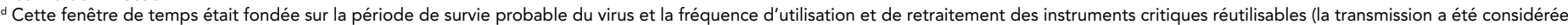
uniquement pour la première personne sur laquelle l'instrument a été utilisé; les cycles de retraitement $\mathrm{n}$ ultérieurs devraient réduire le risque de transmission à nul)

infectiologue par son médecin de famille pour la prise en charge clinique et l'enquête habituelle de santé publique a permis de constater que ce patient présentait d'autres facteurs de risque pour l'infection au VHC (c.-à-d. transmission verticale ou horizontale potentielle par les contacts familiaux). Les dépistages du VHB et du VIH suite au rappel des patients exposés n'ont pas permis d'identifier de nouveaux diagnostics d'infection aiguë ou chronique au VHB ou d'infection au VIH.

Dans l'ensemble, parmi les 4495 patients possiblement exposés à un dispositif médical critique réutilisable à la clinique, selon les données d'analyse de laboratoire transmises à SPO six mois après l'envoi postal de la lettre d'avis aux patients, la prévalence était de $0,07 \%$, IC de $95 \%$ (0 à 2,8 \%) pour l'infection au VHB (chronique ou résolue), de $0,35 \%, I C$ de $95 \%(0,10$ à $0,80 \%)$ pour l'infection au $\mathrm{VHC}$ (chronique ou résolue) et de 0,08 \%, IC de $95 \%$ (0 à 0,49\%) pour l'infection au VIH. Ces résultats sont inférieurs à la prévalence estimée parmi la population canadienne pour le VHB (13), le VHC (14) et le VIH $(15,23)$ et inférieurs aux estimations récentes pour le VHC en Ontario (24).

Parmi les patients diagnostiqués pour la première fois avec une infection au VHB après un acte préoccupant, les risques d'infection au VHB n'étaient pas plus élevés lorsque l'acte s'était déroulé dans les sept jours après un autre acte concernant une personne avec un résultat de dépistage du VHB positif antérieur à son propre acte $\left(R C_{\text {dans les sept jours }}=0,62, I C\right.$ de $95 \%: 0,15$ à 2,63). Parce que les patients ayant subi le dépistage après l'envoi de la lettre d'avis étaient en moyenne plus âgés que ceux n'ayant pas subi d'analyse, un rapport de cotes ajusté selon l'âge a été estimé; l'estimation ajustée selon l'âge était semblable
$\left(R C_{\text {dans les sept jours, ajusté selon l'âge }}=0,59, I C\right.$ de $95 \%: 0,14$ à 2,51). Des résultats statistiquement non significatifs ont également été obtenus en augmentant la fenêtre d'exposition à 28 jours $\left(R C_{\text {dans les } 28 \text { jours, ajusté selon l'âge }}=1,35, I C\right.$ de $95 \%: 0,62$ à 2,93); l'analyse avec la fenêtre d'un jour n'a pas pu être réalisée car le numérateur était égal à zéro. Des résultats semblables ont été obtenus lorsque l'analyse a été réalisée au niveau de l'actepatient $\left(R C_{\text {dans les sept jours, ajusté selon l'âge }}=0,45, I C\right.$ de $95 \%: 0,11$ à 1,85) plutôt qu'au niveau du patient. Il n'a pas été possible d'estimer les risques de transmission du $\mathrm{VHC}$ et du $\mathrm{VIH}$, parce qu'aucun patient n'a d'abord été diagnostiqué avec le $\mathrm{VHC}$ ou le $\mathrm{VIH}$ après avoir subi un acte préoccupant dans les 28 jours suivant un autre acte concernant un patient avec un résultat de dépistage du VHC ou $\mathrm{VIH}$ positif antérieur à son propre acte.

\section{Discussion}

En réaction à une plainte relative aux pratiques de $\mathrm{PCl}, \mathrm{SPO}$ a identifié un manquement aux pratiques de $\mathrm{PCl}$, dont un bris de retraitement des dispositifs médicaux critiques réutilisables, sur quinze ans dans une clinique de médecine familiale. Santé publique Ontario a estimé de manière qualitative que le risque d'infection était «faible " pour le VHB et le VHC, et « très faible " pour le VIH. Les patients possiblement exposés ont été identifiés à l'aide des données de facturation du RASO; ils ont ensuite été avisés individuellement par l'envoi d'une lettre d'avis les informant de leur exposition possible à la suite du bris de RDM et leur recommandant de subir un dépistage pour le VHB, le VHC et le VIH. Six mois après I'envoi des lettres d'avis, seulement $33 \%$ des patients avaient subi un dépistage; 
une proportion plus élevée de patients âgés s'était soumise au dépistage recommandé comparativement aux patients plus jeunes. Le dépistage réalisé suite au bris de RDM a permis $d^{\prime}$ 'identifier deux nouveaux diagnostics d'infection au VHC (c.-à-d. une infection chronique et une infection résolue); le nouveau cas d'infection chronique au VHC aurait probablement été infecté par une transmission verticale ou intrafamiliale. Le dépistage suite au bris de RDM n'a pas révélé de nouveaux diagnostics d'infection au VHB ou au VIH. La prévalence de I'infection au VHB, VHC et VIH parmi les patients possiblement exposés était inférieure à celle de la population canadienne. Le risque d'infection au VHB n'était pas plus élevé parmi les patients ayant subi un acte préoccupant dans les 7 ou 28 jours suivant un autre acte concernant un patient avec un résultat de VHB positif antérieur à son propre acte. II n'a pas été possible d'estimer le risque d'infection au VHC et au VIH parce que les nombres étaient insuffisants. L'enquête de Santé publique Ottawa n'a révélé aucune évidence de transmission du VHB, du VHC ou du VIH associée au bris de RDM. Cependant, on ne peut pas complètement exclure la possibilité d'une transmission, puisque seulement le tiers des patients possiblement exposés ont subi les tests de dépistage recommandés.

L'enquête de SPO en lien avec un bris de RDM à une clinique d'endoscopie en 2011, qui avait concerné 6992 patients (17), parmi lesquels $75 \%$ s'étaient soumis au dépistage du VHB, VHC et VIH, n'avait pas non plus identifié de transmission. De plus, une revue des éclosions de VHB et VHC associées aux soins de santé déclarées au CDC des États-Unis n'a permis d'identifier aucune éclosion de VHB ou de $\mathrm{VHC}$ reliée avec un bris de retraitement de dispositifs médicaux critiques réutilisables semblables à ceux concernés par le présent bris de RDM (c.-à-d. des dispositifs médicaux utilisés pour effectuer des interventions chirurgicales mineures) (1). Les écarts entre les pratiques observées et les pratiques exemplaires en matière de $\mathrm{PCl}$ associés avec une transmission du VHB et du VHC étaient plutôt liés aux analyses de glycémie au chevet du patient et à la mauvaise utilisation des flacons multidoses et du matériel d'injection (1).

\section{Points forts et limites}

Un point fort important de cette enquête était la capacité d'identifier les patients possiblement exposés à partir des données de facturation du RASO. Ce processus pourrait être amélioré si les données du RASO comprenaient un identifiant unique obligatoire pour le milieu de soins où l'acte a été effectué. D'autres points forts comprenaient la centralisation des analyses de dépistage au LSPO, qui a permis de faire le suivi des résultats, ainsi que l'accès aux résultats des analyses de laboratoire précédentes pour identifier les patients possiblement exposés avec un résultat antérieur positif pour le VHB, le VHC ou le $\mathrm{VIH}$. Une limite importante de cette enquête était la participation relativement faible au dépistage et le biais de participation potentiel parce que les patients choisissaient euxmêmes de subir ou non le dépistage. Les patients qui ont choisi de subir le dépistage étaient en moyenne plus âgés que ceux qui ne l'ont pas fait; nous avons tenté d'ajuster les résultats de nos analyses pour cette différence en estimant un RC ajusté selon I'âge, qui s'est avéré semblable au RC non-ajusté. À l'exception de l'âge (c.-à-d. la date de naissance), les caractéristiques sociodémographiques des patients potentiellement exposés au bris de RDM n'étaient pas recueillies ni disponibles, et nous n'avons pas pu évaluer si d'autres facteurs étaient associés à la participation au dépistage. De plus, plusieurs facteurs limitaient notre capacité à vérifier l'état d'exposition, ce qui aurait pu mener à une erreur de classification de l'exposition et à une dilution de l'effet : I'incapacité de déterminer quel patient avait été exposé à un instrument donné, parce qu'il n'y avait pas de système de traçabilité des instruments (p. ex. code à barres) en place à cette clinique, le manque d'accès aux résultats négatifs pour le $\mathrm{VHB}$, le $\mathrm{VHC}$ et le $\mathrm{VIH}$ pour tout test effectué avant le rappel des patients (à l'exception des patients avec un résultat positif pour la même infection, parce que les résultats négatifs n'ont pas à être déclarés à la santé publique), le manque d'information concernant le moment précis auquel les patients ont été infectés par le VHB, VHC ou VIH, et le manque d'information concernant la contagiosité au moment de l'acte préoccupant.

\section{Conclusion}

Il est important de partager les résultats des enquêtes à grande échelle suite aux bris de RDM pour que ces résultats puissent contribuer à orienter l'intervention de santé publique lors de futurs bris semblables. Les enquêtes sur les bris de RDM à venir pourraient tirer profit d'une meilleure évaluation du statut infectieux et de l'exposition, par exemple, en ayant accès à l'ensemble des résultats de dépistage du VHB, VHC et $\mathrm{VIH}$ (y compris les résultats négatifs) contenus dans les bases de données des laboratoires. De plus, puisque l'évaluation du risque d'infection au $\mathrm{VHB}, \mathrm{VHC}$ et $\mathrm{VIH}$ associé à un bris de RDM constitue un processus imprécis et ce, que l'on utilise une méthode d'évaluation du risque qualitative $(25,26)$ ou quantitative $(11,12)$, une surveillance provinciale des bris de RDM et des interventions de santé publique en lien avec ceux-ci, ainsi que la prise en compte des résultats de ces enquêtes permettraient de renforcer les données probantes qui sous-tendent les évaluations du risque et aiderait au processus décisionnel concernant le rappel et le dépistage des patients.

En Ontario, la prévention des manquements aux pratiques de $\mathrm{PCl}$ excède en grande partie le mandat officiel des unités de santé publique; cette responsabilité est surtout celle des gestionnaires des cliniques et fournisseurs de services, des programmes de formation des professionnels de la santé, des organismes d'attribution des permis, des ordres professionnels et du ministère de la Santé et des Soins de longue durée de I'Ontario. Cela dit, après ce bris de RDM, SPO s'est associé à l'Ordre des médecins et chirurgiens de l'Ontario et à Santé publique Ontario pour offrir une séance de formation professionnelle continue sur les pratiques exemplaires en matière de $\mathrm{PCl}$ à l'intention des médecins de famille d'Ottawa. De plus, SPO a développé et livré une séance de formation pour 
5. Ontario Ministry of Health and Long-Term Care. Ontario Public Health Standards: Requirements for Programs,

les résidents en médecine familiale de l'Université d'Ottawa. Enfin, SPO a évalué les pratiques actuelles et les besoins $\mathrm{d}$ 'apprentissage en matière de $\mathrm{PCl}$ des cliniques médicales et dentaires d'Ottawa (27) dans le but d'orienter de futures interventions visant à améliorer les pratiques de $\mathrm{PCl}$ dans ces milieux.

\section{Déclaration des auteurs}

Cadieux était l'auteur principal du manuscrit. Friedman a contribué à l'évaluation de l'exposition et du résultat, en plus de fournir de la rétroaction sur le manuscrit. Tilley a réalisé l'évaluation de l'exposition et du résultat, en plus de fournir de la rétroaction sur le manuscrit. Mazzulli a supervisé les analyses de laboratoire et a contribué au manuscrit. McDermaid a réalisé les analyses statistiques et a contribué au manuscrit.

\section{Conflit d'intérêts}

Aucun.

\section{Remerciements}

Les auteurs souhaitent remercier les personnes et organismes ayant participé à l'enquête sur le bris de retraitement des dispositifs médicaux, ainsi que les personnes et collectivités touchées par ce bris.

\section{Financement}

Les auteurs ont reçu une aide salariale de leurs organismes respectifs pour réaliser le présent travail.

\section{Références}

1. Centers for Disease Control and Prevention. Viral Hepatitis. Atlanta (GA): CDC; 2018 (Accédé 2019-0427). https://www.cdc.gov/hepatitis/outbreaks/pdfs/ healthcareinvestigationtable.pdf

2. Cadieux G, Brown C, Sachdeva H. Enquête des plaintes relatives aux pratiques de prévention et de contrôle des infections par la santé publique en Ontario, de 2015 à 2018. Relevé des maladies transmissibles au Canada 2019;45(11):318-25. DOI

3. Ontario Health Protection and Promotion Act, R.S.O. 1990, c. H.7. Ontario; 1990. https://www.ontario.ca/laws/ statute/90h07/v6

4. Association of Local Public Health Agencies. Milestones and History: History of Public Health Units in Ontario. Toronto (ON): alPHa; 2018 (Accédé 2019-10-29).

https://www.alphaweb.org/page/milestones
Services, and Accountability (Standards). Gov't of Ontario; 2018 (Accédé 2019-09-07). http://www.health.gov.on.ca/en/ pro/programs/publichealth/oph_standards/docs/protocols_ guidelines/IPAC_Complaint_Protocol_2019_en.pdf

6. Ontario Ministry of Health and Long-Term Care. Infection Prevention and Control Complaint Protocol, 2019. Gov't of Ontario; 2019 (Accédé 2019-09-07). http://www.health. gov.on.ca/en/pro/programs/publichealth/oph_standards/ docs/protocols_guidelines/IPAC_Complaint_Protocol_2019_ en.pdf

7. Public Health Ontario. IPAC Checklist for Clinical Office Practice: Core Elements. PHO; 2018 (Accédé 2019-09-07). https://www.publichealthontario.ca/-/media/documents/ checklist-clinical-office-core.pdf?la=en

8. Provincial Infectious Diseases Advisory Committee. (2013). Best Practices for Cleaning, Disinfection and Sterilization of Medical Equipment/Devices In All Health Care Settings, 3rd edition (Accédé 2019-09-07). https://www. publichealthontario.ca/-/media/documents/bp-cleaningdisinfection-sterilization-hcs.pdf?la=en

9. Provincial Infectious Diseases Advisory Committee. (2015). Infection Prevention and Control for Clinical Office Practice (Accessed: 2019-09-07). https://www. publichealthontario. $\mathrm{ca} /$-/media/documents/bp-clinical-office-practice.pdf?la=en

10. Ontario Ministry of Health and Long-Term Care. (2019). Infection Prevention and Control Disclosure Protocol (Accédé 2019-09-07). http://www.health.gov.on.ca/en/pro/ programs/publichealth/oph_standards/docs/protocols_ guidelines/Infection_Prevention_and_Control_Disclosure_ Protocol_2019_en.pdf

11. Weber DJ, Rutala WA. Assessing the risk of disease transmission to patients when there is a failure to follow recommended disinfection and sterilization guidelines. Am J Infect Control 2013 May;41(5 Suppl):S67-71. DOI PubMed

12. Rutala WA, Weber DJ. How to assess risk of disease transmission to patients when there is a failure to follow recommended disinfection and sterilization guidelines. Infect Control Hosp Epidemiol 2007 Feb;28(2):146-55.

DOI PubMed

13. Rotermann M, Langlois $K$, Andonov A, Trubnikov M. Seroprevalence of hepatitis $B$ and $C$ virus infections: Results from the 2007 to 2009 and 2009 to 2011 Canadian Health Measures Survey. Health Rep 2013 Nov;24(11):3-13. PubMed

14. Trubnikov M, Yan $P$, Archibald $C$. Estimation de la prévalence de l'infection par le virus de l'hépatite $\mathrm{C}$ au Canada, 2011. Relevé des maladies transmissibles au Canada 2014;40(19):442 -50. DOI 
15. Agence de la santé publique du Canada. Résumé : Estimations de l'incidence de la prévalence, et de la proportion non diagnostiquée au VIH au Canada, 2014. Ottawa (ON) : ASPC; 2015 (Accédé 2019-1029). https://www.canada.ca/content/dam/canada/ health-canada/migration/healthy-canadians/publications/ diseases-conditions-maladies-affections/hiv-aidsestimates-2014-vih-sida-estimations/alt/hiv-aids-estimates2014-vih-sida-estimations-fra.pdf

16. U.S. Public Health Service. Updated U.S. Public Health Service Guidelines for the Management of Occupational Exposures to HBV, HCV, and HIV and Recommendations for Postexposure Prophylaxis. MMWR Recomm Rep 2001 Jun;50 RR-11:1-52. PubMed

17. Willmore J, Ellis E, Etches $V$, Labrecque L, Osiowy $C$, Andonov A, McDermaid C, Majury A, Achonu C, Maher M, MacLean B, Levy I. Public health response to a large-scale endoscopy infection control lapse in a nonhospital clinic. Can $\mathrm{J}$ Infect Dis Med Microbiol 2015 Mar-Apr;26(2):77-84. DOI PubMed

18. Bond WW, Favero MS, Petersen NJ, Gravelle CR, Ebert JW, Maynard JE. Survival of hepatitis B virus after drying and storage for one week. Lancet 1981 Mar;1(8219):550-1. DOI PubMed

19. Ciesek S, Friesland M, Steinmann J, Becker B, Wedemeyer H, Manns MP, Steinmann J, Pietschmann T, Steinmann E. How stable is the hepatitis $\mathrm{C}$ virus (HCV)? Environmental stability of HCV and its susceptibility to chemical biocides. J Infect Dis 2010 Jun;201(12):1859-66. DOl PubMed

20. Doerrbecker J, Friesland M, Ciesek S, Erichsen TJ, Mateu-Gelabert P, Steinmann J, Steinmann J, Pietschmann T, Steinmann E. Inactivation and survival of hepatitis $C$ virus on inanimate surfaces. J Infect Dis 2011 Dec;204(12):1830-8. DOI PubMed
21. Kamili S, Krawczynski K, McCaustland K, Li X, Alter MJ. Infectivity of hepatitis $C$ virus in plasma after drying and storing at room temperature. Infect Control Hosp Epidemiol 2007 May;28(5):519-24. DOI PubMed

22. Paintsil E, Binka M, Patel A, Lindenbach BD, Heimer R. Hepatitis $C$ virus maintains infectivity for weeks after drying on inanimate surfaces at room temperature: implications for risks of transmission. J Infect Dis 2014 Apr;209(8):1205-11. DOI PubMed

23. Agence de la santé publique du Canada. Résumé : Estimations de l'incidence et de la prévalence du VIH, et des progrès réalisés par le Canada en ce qui concerne les cibles 90-90-90 pour le VIH, 2016. Ottawa (ON) : PHAC; 2018 (Accédé on: 2019-11-09). https://www.canada.ca/ content/dam/phac-aspc/documents/services/publications/ diseases-conditions/summary-estimates-hiv-incidenc e-prevalence-canadas-progress-90-90-90/pub-fra.pdf

24. Bolotin S, Feld JJ, Garber G, Wong WW, Guerra FM, Mazzulli T. Population-based estimate of hepatitis C virus prevalence in Ontario, Canada. PLoS One 2018 Jan;13(1):e0191184. DOI PubMed

25. Patel PR, Srinivasan A, Perz JF. Developing a broader approach to management of infection control breaches in health care settings. Am J Infect Control 2008 Dec;36(10):685-90. DOI PubMed

26. Centers for Disease Control and Prevention. Steps for Evaluating an Infection Control Breach. Atlanta (GA); CDC; 2012 (Accédé 2019-04-27). https://www.cdc.gov/hai/ outbreaks/steps_for_eval_ic_breach.html

27. Cadieux G, Bhatnagar A, Schindeler T, Prematunge C, Perron D, Willmore J. Assessment of the infection prevention and control learning needs of Ottawa community-based healthcare providers. Can J Infect Control 2019;34(3):135-40. https://www.researchgate.net/publication/337735988 